\title{
Invited Discussion on: The Effect of Two Different Frontalis Sling Approaches on Postoperative Eyelid Contour: A Comparative Study
}

\author{
Catherine J. Hwang ${ }^{1}$
}

Received: 26 January 2020/ Accepted: 27 January 2020/Published online: 13 February 2020

(C) Springer Science+Business Media, LLC, part of Springer Nature and International Society of Aesthetic Plastic Surgery 2020

Level of Evidence $V$ This journal requires that authors assign a level of evidence to each article. For a full description of these Evidence-Based Medicine ratings, please refer to the Table of Contents or the online Instructions to Authors www.springer.com/00266.

Frontalis sling correction for congenital ptosis remains challenging as it often has eyelid height and contour issues. In this study, the authors compare eyelid contour after orbicularis muscle fixation and sutured tarsal fixation with lid crease formation using photographs to measure eyelid height at three positions.

The eyelid heights at each of the three measurements (nasal, central, and temporal) were similar in both surgical groups, but various ratios of the measurements in the tarsal fixation group were closer to those of the normal population, implying tarsal fixation allowed for a more natural eyelid contour.

Several methodological flaws limit interpretation of the data. The two groups had different rates of unilateral versus bilateral ptosis and different gender mixes. The suture fixation group underwent additional surgery (crease formation) that could have affected results. The authors compared contours of operated eyes to a normal control group rather than the contralateral unoperated eye in unilateral cases. The control group was also not matched for age, ethnicity, or gender. The $N$ was small and follow-up was less than 3 months in some patients. This is particularly unfortunate because it would seem the suture fixation may yield better contour over time as the non-fixated sling

Catherine J. Hwang

Hwangc2@ccf.org

1 Cole Eye Institute, Cleveland Clinic Foundation, 9500 Euclid Ave, Cleveland, OH 44195, USA erodes through the eyelid tissues, but the study did not test for this possibility.

The authors should be congratulated for attempting to find a new method to measure eyelid contour, as no current system allows for a quick way to clinically capture this important outcome measure. However, the authors' system has not been validated, and it still requires painstaking measurements of digital photographs. More importantly, the system may not be as accurate, comparable, or reproducible as other previously published systems. They use the pupil margin as the inferior measure, but the pupil margin is a changing target, unlike the marginal reflex distance. They also only measure the eyelid height at only three points, which is not enough to characterize the curvature of the entire eyelid, only the central $1 / 3$ of the eyelid.

The authors also don't discuss whether patients were using the frontalis muscle, which is critical in these cases because the entire success of the operation depends on the ability to use the frontalis muscle to lift the eyelid.

Given these methodological flaws, the results are not compelling enough for me to perform tarsal fixation of silicone slings in every case. The surgical approach and material used for congenital ptosis is a complex decision. It makes sense to perform suture fixation of the sling when a crease is being formed, as the area is already directly exposed with a separate incision. However, minimally invasive techniques, i.e., those without eyelid crease formation, are also desired to minimize scar tissue formation and preserve orbicularis function, especially because these patients may require multiple surgeries. In these cases, I would not create a separate incision based on the results of this study.

In addition to the two surgical techniques used in this study, non-rhomboid configuration techniques exist that 
can avoid the central brow stab incision. It would be nice to see the long-term data comparing various surgical techniques and materials in relation to the contour, how the contour may change over time, and to see when failure or cheesewiring of the sling may occur and whether suturing may prevent this.

It was a pleasure reading the authors' attempts to measure and improve contour for treatment of congenital ptosis, and their work provides an additional foundation to help us improve our congenital ptosis repair results.

\section{Compliance with Ethical Standards}

Conflict of interest The author declares that she has no conflicts of interest to disclose.

Publisher's Note Springer Nature remains neutral with regard to jurisdictional claims in published maps and institutional affiliations. 